\title{
2D/3D reconstruction of the distal femur using statistical shape models addressing personalized surgical instruments in knee arthroplasty: A feasibility analysis
}

\author{
Pietro Cerveri ${ }^{1}$ | Costanza Sacco ${ }^{1}$ | Gianluca Olgiati ${ }^{2}$ | Alfonso Manzotti ${ }^{3}$ | Guido Baroni ${ }^{1}$
}

\footnotetext{
${ }^{1}$ Department of Electronics, Information and Bioengineering, Politecnico di Milano University, Milan, Italy

${ }^{2}$ Medacta International SA, Castel San Pietro, Switzerland

${ }^{3}$ Orthopaedic and Trauma Department, "Luigi Sacco" Hospital, ASST FBF-Sacco, Milan, Italy

Correspondence

Pietro Cerveri, Department of Electronics, Information and Bioengineering, Politecnico di Milano University - Milan, Italy.

Email: pietro.cerveri@polimi.it
}

\begin{abstract}
Background: Personalized surgical instruments (PSI) have gained success in the domain of total knee replacement, demonstrating clinical outcomes similar or even superior to both traditional and navigated surgeries. The key requirement for prototyping PSI is the availability of the digital bony surface. In this paper, we aim at verifying whether the 2D/3D reconstruction of the distal femur, based on statistical shape models (SSM), grants sufficient accuracy, especially in the condylar regions, to support a PSI technique.
\end{abstract}

Methods: Computed tomographic knee datasets acquired on 100 patients with severe cartilage damage were retrospectively considered in this work. All the patients underwent total knee replacement using the PSI-based surgical technique. Eighty out of 100 reconstructed distal femur surfaces were used to build the statistical model. The remaining 20 surfaces were used for testing. The 2D/3D reconstruction process was based on digital reconstructed radiographies (DRRs) obtained with a simulated X-ray projection process. An iterative optimization procedure, based on an evolutionary algorithm, systematically morphed the statistical model to decrease the difference between the DRR, obtained by the original CT dataset, and the DRR obtained from the morphed surface.

Results: Over the 80 variations, the first ten modes were found sufficient to reconstruct the distal femur surface with accuracy. Using three DRR, the maximum Hausdorff and RMS distance errors were lower than 1.50 and $0.75 \mathrm{~mm}$, respectively. As expected, the reconstruction quality improved by increasing the number of DRRs. Statistical difference $(P<0.001)$ was found in the 2 vs 3,2 vs 4 and 2 vs 5 DRR, thus proving that adding just a single displaced projection to the two traditional sagittal and coronal X-ray images improved significantly the reconstruction quality. The effect of the PSI contact area errors on the distal cut direction featured a maximum median error lower than $2^{\circ}$ and $0.5^{\circ}$ on the sagittal and frontal plane, respectively. Statistical difference was found $(P<0.0001)$ in the reconstruction accuracy when comparing SSM built using pathologic with respect to non-pathologic shapes (cadavers), meaning that, to improve the patient-specific reconstruction, the morphologic anomalies, specific to the pathology, must be embedded into the SSM.

Conclusions: We showed that the $\mathrm{X}$-ray based reconstruction of the distal femur is reasonable also in presence of pathologic bony conditions, featuring accuracy results similar to earlier reports in the literature that reconstructed normal femurs. This finding discloses the chance of applying the proposed methodology to the reconstruction of bony surfaces used in the PSI surgical approach.

\section{KEYWORDS}

CT scans, digital reconstructed radiography, distal femur, knee arthroplasty, osteophytes, statistical shape model

This is the peer reviewed version of the following article: "2D/3D reconstruction of the distal femur using statistical shape models addressing personalized surgical instruments in knee arthroplasty: A feasibility analysis", which has been published in final form at http://dx.doi.org/10.1002\%2Frcs.1823. This article may be used for non-commercial purposes in accordance with Wiley Terms and Conditions for Use of Self-Archived Versions 


\section{1 | INTRODUCTION}

Personalized surgical instruments (PSI) have gained success in the domain of total knee replacement, proving clinical outcomes comparable or even superior to both conventional and navigated surgical techniques. ${ }^{1-4}$ Basically PSI are disposable cutting blocks that match the outer shape of a patient's distal femur and proximal tibia, allowing surgeons to make the precise bone cuts needed to position the knee implant at the optimal alignment according to the computer-based planning. ${ }^{5}$ The PSI technique, accepted for advanced osteoarthritis, severe discomfort, and reduced function/walking capability, spares the intramedullary canals, exploited instead with the conventional instruments, and does not require invasive intra-osseous pins, which are mandatory in the navigated surgery. ${ }^{6,7}$ While questioning costeffectiveness, ${ }^{8,9}$ different studies described the advantages of PSI surgery as a more reproducible neutral postoperative alignment and decreased surgical time, with no further intra-operative complications. ${ }^{10,11}$ Despite the benefit of being less invasive, the use of PSI requires the availability of the digital bony surfaces, which are obtained from pre-operative computed tomography (CT) or magnetic resonance imaging (MRI), the identification of anatomical landmarks to allow the planning and design of the personalized resection instruments. ${ }^{12,13}$ Morphologic inaccuracies in the digital description of the bones can reflect on planned PSI matching and consequently on the implant size choice and alignment setup. ${ }^{7,10}$

Volumetric scans have the benefit of providing the complete three-dimensional (3D) description of the anatomy at the price of being expensive and/or distressing for the patients due to additional radiation doses. In order to address both issues, statistical shape models (SSM) and multiple calibrated radiographic images have been taken into account as a valuable alternative to the reconstruction of patient-specific 3D surface models. ${ }^{14-20}$ The main advantage of SSM, with respect to other reconstruction and surface modeling approaches, ${ }^{21}$ is that they describe the morphologic features of a set of surface model prototypes, which constitute the training dataset of the SSM, synthetically into a space of fundamental modes of variations. ${ }^{14,15}$ The construction working principle relies on the iterative 3D morphing (surface deformation) of the SSM aiming at maximizing the $2 \mathrm{D}$ matching between the digitally reconstructed radiographies (DRR), obtained from the virtual binary volume of the morphed SSM, and the corresponding real radiographies acquired on the patient ${ }^{19,20}$ (Figure 1 ). In this 2D/3D reconstruction method using SSM, two main factors affect the quality of the results, namely the extrapolation property of the SSM, and the number and orientation of the DRRs. As far as SSM extrapolation property is concerned, it is well known that the quality of the morphing depends upon the morphologic variability encompassed in the training dataset. ${ }^{22}$ For orthopedic applications, this means that pathologic bony anomalies, unless sufficiently represented in the training dataset, turn out to be hardly reproducible in the reconstruction process. As far as DRRs are concerned, both single and multiple $\mathrm{X}$-ray projections have been proposed, ${ }^{23-25}$ but no systematic analysis focused on optimizing the DRR setup to improve the reconstruction accuracy and ensure the reproduction of local morphologic anomalies. For instance, the accuracy of the condylar osteophyte reconstruction, namely bony spurs that develops in severe osteoarthrosis, would benefit from axial radiographies in addition to conventional frontal and coronal views. However, it is obvious that axial X-ray projections are unviable, thus requiring a careful evaluation of alternative orientations. With the recent mobile C-Arm X-ray systems, the acquisition of a number of calibrated $\mathrm{X}$-ray projections appears to be feasible so that SSM can be considered a fruitful option for 3D surface reconstruction. ${ }^{26}$

In the light of this premise, this paper aims at evaluating whether the $2 D / 3 D$ reconstruction, based on morphing a distal femur SSM to match the projective information of X-ray images, is reliable for PSIbased surgical planning. The study involved a dataset of CT scans, along with the corresponding reconstructed surfaces, from 100 patients affected by severe cartilage damage, who underwent total

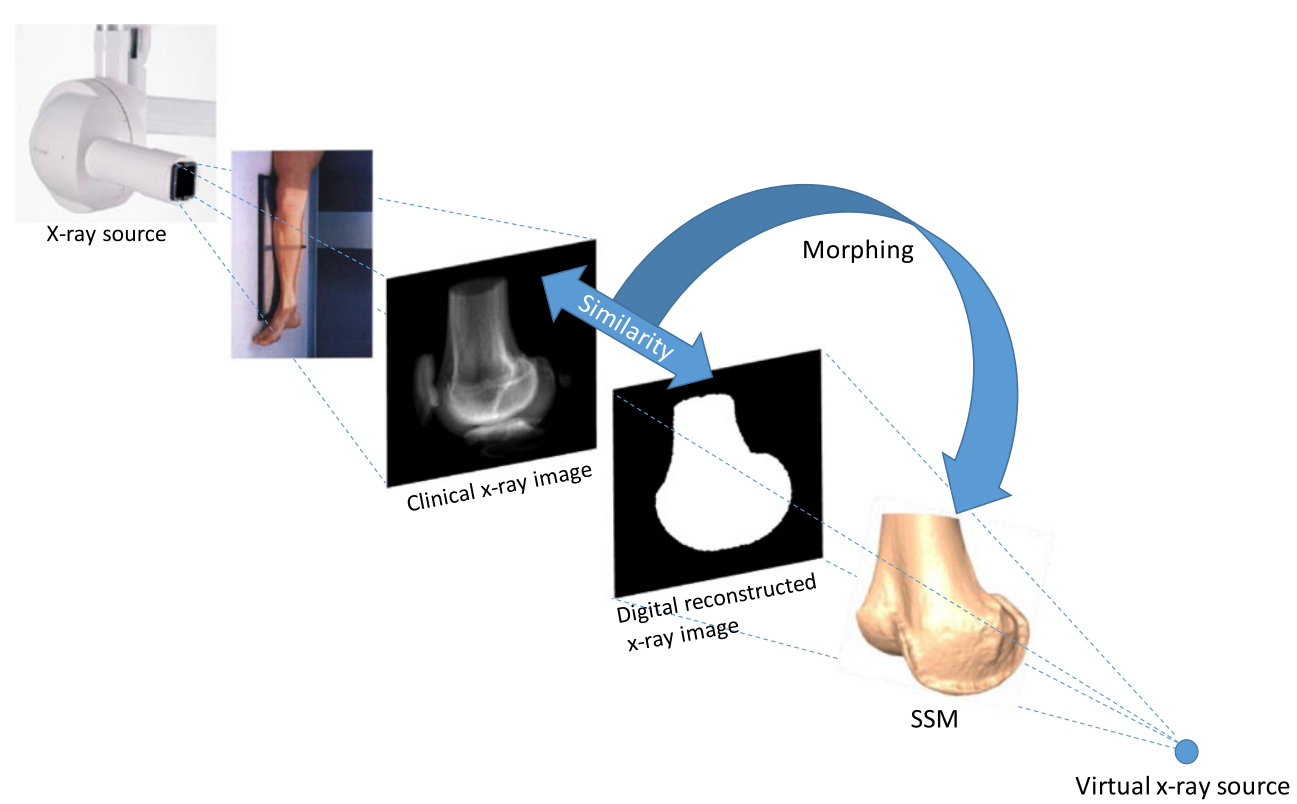

FIGURE 1 Basic general schema of reconstruction using statistical shape models by mean of X-ray images 
knee replacement using the PSI-based surgical technique. The reconstruction quality was tested with respect to the number of SSM parameters, the number of DRRs, and use of off-the-axial-plane DRRs. We analyzed the spatial localization of the errors focusing on the distal condylar regions and verified the deviation of the distal cut from the planned direction because of PSI contact areas errors. In order to investigate the ability to reproduce condylar osteophytes, we compared the reconstructions obtained using the pathologic SSM with those obtained using one SSM built on a cadaveric dataset of 20 non-pathologic distal femurs.

\section{2 | MATERIALS AND METHODS}

\section{1 | Statistical bone model and surface morphing}

The SSM representation relies on the decomposition of the morphologic differences featured in the shape dataset by applying principal component analysis (PCA). These shapes form the training dataset by means of which SSM learns the mean shape $\bar{s}$ (the most probable shape in the set) and the covariance matrix $\mathbf{C}$ of the differences of each shape in the set with respect to $\bar{s}$. Explicitly, the mean shape is an averaged morphologic representation of the training set while matrix $C$ encodes the principal modes of variation about $\bar{s}$, namely the morphologic dissimilarities in the training dataset. Given that the training set consists of $M$ surfaces, each featuring $N$ points in 3D space, the PCA determines an orthonormal basis of $M$ directions (eigenvectors) with its axes sorted to encode most to least relevant modes of variation. Explicitly, the first axis is oriented along the direction that quantifies the highest standard deviation $\sigma_{1}$ (squared eigenvalues). All the following directions, subjected to the constraint that they must be orthogonal to the preceding axes, account for progressively decreasing standard deviations $\sigma_{j}$ up to $j=M$. Eigenvectors $\left\{v_{j}\right\}$ and the corresponding eigenvalues $\left\{\sigma_{j}^{2}\right\}$ are calculated from the covariance matrix $\mathrm{C}$ by Eigen decomposition. Vectors $\left\{\mathrm{v}_{j}\right\}$ are the principal directions spanning the shape space with $\bar{s}$ representing its origin. Hence, each mode of variation $j$ in the PCA can be regarded as a normal distribution featuring variance $\sigma_{j}^{2}$.

Surface models of the distal femur, obtained by segmenting CT datasets of 47 left and 53 right knees from a patient population aged $67 \pm 10$ years, were considered in this study. All the patients, affected by severe cartilage damage, underwent total knee replacement surgery by means of MyKnee ${ }^{\mathrm{TM}}$ PSI (Medacta, $\mathrm{CH}$ ). Some 520 axial slices were available for each patient. The image resolution and the voxel size of the CT scans were $512 \times 512$ and $0.48 \times 0.48 \times 0.5 \mathrm{~mm}$, respectively. Expert radiological operators performed the image segmentation manually. Orthopedic surgeons revised the corresponding cases before PSI prototyping. The pre-operatory hip-knee-ankle and tibial slope angles were on average $176^{\circ}$ and $9^{\circ}$, respectively.

After automatic reconstruction, each surface model, described by a triangle mesh, was down-sampled to some 8000 vertices. Left femurs (both CT volumes and surfaces) were mirrored in medio-lateral direction to obtain an overall dataset of 100 right distal femurs. Most cases showed evident bony anomalies especially at condylar level (Figure 2).

The SSM model was built by randomly choosing 80 surfaces in the dataset of the 100 surfaces. In order to compute $\bar{s}$, an automatic custom procedure was developed that computed point-to-point correspondences across the 80 surfaces. First, one surface was randomly selected in the dataset to serve as reference for alignment. In order to co-register the remaining surfaces to the reference surface, the point coherent drift algorithm (CPD), based on deformable registration, was used. ${ }^{27}$ Prior to deformation, all the surfaces underwent rigid registration to recover major differences in the position and attitude. ${ }^{28}$ After alignment, the point correspondences were automatically determined and the mean shape computed using the rigidly registered surfaces. Across the 80 shapes, $N=6000$ valid correspondences were found. The covariance matrix was computed from the matrix
FIGURE 2 One right knee (patient 94) in the study dataset. The reconstructed surface was superimposed in the lateral, axial and coronal views to the corresponding CT scan. Large bony deformations are apparent on the frontal aspect of the trochlear region, the medial facet of the trochlear region and both medial and lateral distal regions of the two condyles

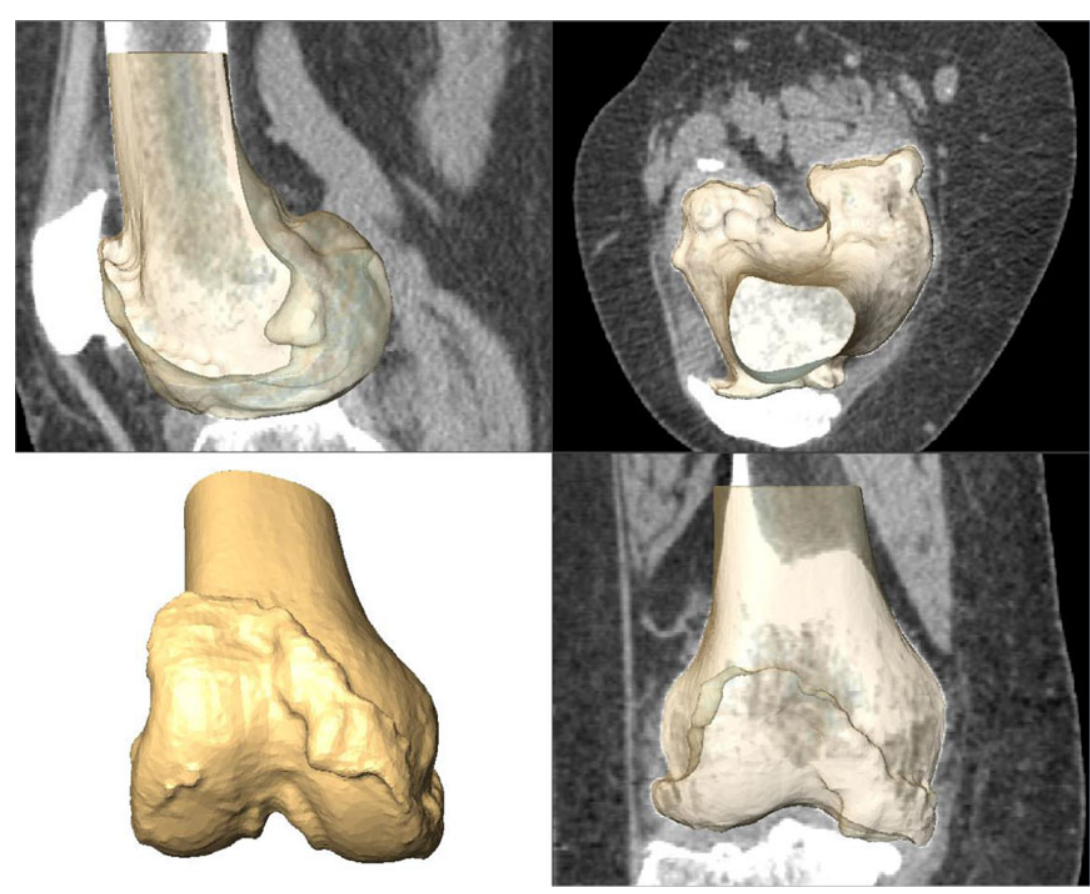


difference $D(3 N \times M)$, which describes the coordinate variations of the points of the surfaces with respect to $\bar{s}$, as:

$$
C=D^{T} D \text { with } D=\left[\begin{array}{ccc}
x_{1,1}-\bar{x}_{1} & . . & x_{1, M}-\bar{x}_{1} \\
y_{1,1}-\bar{y}_{1} & . . & x_{1, M}-\bar{x}_{1} \\
z_{1,1}-\bar{z}_{1} & . . & x_{1, M}-\bar{x}_{1} \\
. . & x_{i, j}-\bar{x}_{i} & . . \\
. . & y_{i, j}-\bar{y}_{i} & . . \\
. . & z_{i, j}-\bar{z}_{i} & . . \\
x_{N, 1}-\bar{x}_{N} & . . & x_{N, M}-\bar{x}_{N} \\
y_{N, 1}-\bar{y}_{N} & . . & y_{N, M}-\bar{y}_{N} \\
z_{N, 1}-z_{N} & . . & z_{N, M}-\bar{z}_{N}
\end{array}\right]
$$

where $i$ and $j$ account for the ith point in the $j$ th surface model (Figure 3) whereas $\left(\bar{x}_{i}, \bar{y}_{i}, \bar{z}_{i}\right)$ is the generic point of the mean shape. According to the reconstruction based on PCA, the morphing process generates a new surface $s$ by adding to $\bar{s}$ the weighted summation of the eigenvectors, scaled by the corresponding eigenvalues over all the modes of variations $M$ as:

$$
s=\bar{s}+\sum_{j=1}^{M} \alpha_{j} \sigma_{j} v_{j}
$$

where the $\left\{a_{j}\right\}$ parameters modulate the morphing. Assuming Gaussian distribution for each variation mode, it was pointed out that the morphing result is reasonable if $-3<\alpha_{j}<+3$ holds. ${ }^{18}$ The advantage of using PCA is that the sorted Eigen-modes account for decreasing levels of shape dissimilarities thus granting that the contribution of one eigenvector becomes more and more negligible as the corresponding eigenvalue approaches zero. Mathematically this can be quantified by using the concept of explained variance $e_{\sigma_{j}^{2}}$, which correlates each eigenvalue with a specific percentage of variation into the training dataset, computed as:

$$
e_{\sigma_{j}^{2}}=\frac{\sigma_{j}^{2}}{\sum_{i=1}^{M} \sigma_{i}^{2}} \times 100
$$

so that summing up all the Eigen-modes equals $100 \%$. By enduring a reduction of the extrapolation ability, while acceptable, it is possible to represent the morphing by a reduced number $(H<M)$ of variation modes, which accumulate most of the contributions of the dataset dissimilarities.

\section{2 | DRR and image processing}

The radiographic-based reconstruction process aims at determining the parameters $\left\{a_{j}\right\}$ to morph the mean shape in such a way that its digital reconstructed radiographies optimally match the corresponding patient X-ray images. According to a predefined projection geometry setup, the DRR of the morphed surface were obtained by applying the raytracing algorithm to the CT-equivalent volume. ${ }^{29} \mathrm{CT}$-equivalent volume was computed by casting each surface vertex $\left(x_{v}, y_{v}, z_{v}\right)$ to the couple of integer values $\left(i_{u}, j_{u}, k_{u}\right)$ and $\left(i_{a}, j_{a}, k_{a}\right)$, which are actually voxel coordinates, by:

$$
\begin{gathered}
x_{v}=\frac{\left(x-o_{x}\right)}{s_{x}} \quad y_{v}=\frac{\left(y-o_{y}\right)}{s_{y}} \quad z_{v}=\frac{\left(z-o_{z}\right)}{s_{z}} \\
i_{u}=\operatorname{ceil}\left(x_{v}\right) i_{a}=\text { floor }\left(x_{v}\right) \\
j_{u}=\operatorname{ceil}\left(y_{v}\right) j_{a}=\text { floor }\left(y_{v}\right) \\
k_{u}=\operatorname{ceil}\left(z_{v}\right) k_{a}=\text { floor }\left(z_{v}\right)
\end{gathered}
$$

where $\left(o_{x}, o_{y}, o_{z}\right)$ and $\left(s_{x}, s_{y}, s_{z}\right)$ are the volume offset and the voxel size, respectively. As we performed simulated reconstruction, patient $X$-ray projections were replaced by the DRR of the patient $C T$ volume obtained by using the same projection geometry setup (Figure 4). In order to emphasize the difference between the two DRR sets, the first one was called sDRR, namely obtained from the CT-equivalent volume of the morphed surface, and vDRR, the one obtained from the patient $\mathrm{CT}$ volume.

Before projection matching takes place, DRR underwent a specific processing to extract the contours. As SDRR featured sparse pixel data (Figure 4(B)), the image was made binary first and filtered by applying a Gabor filter (pixels/cycle [2 3], wavelengths: [-45,45]). ${ }^{30}$ A Canny edge detector was then applied (Figure $4(C))$. As far as vDRR are concerned,

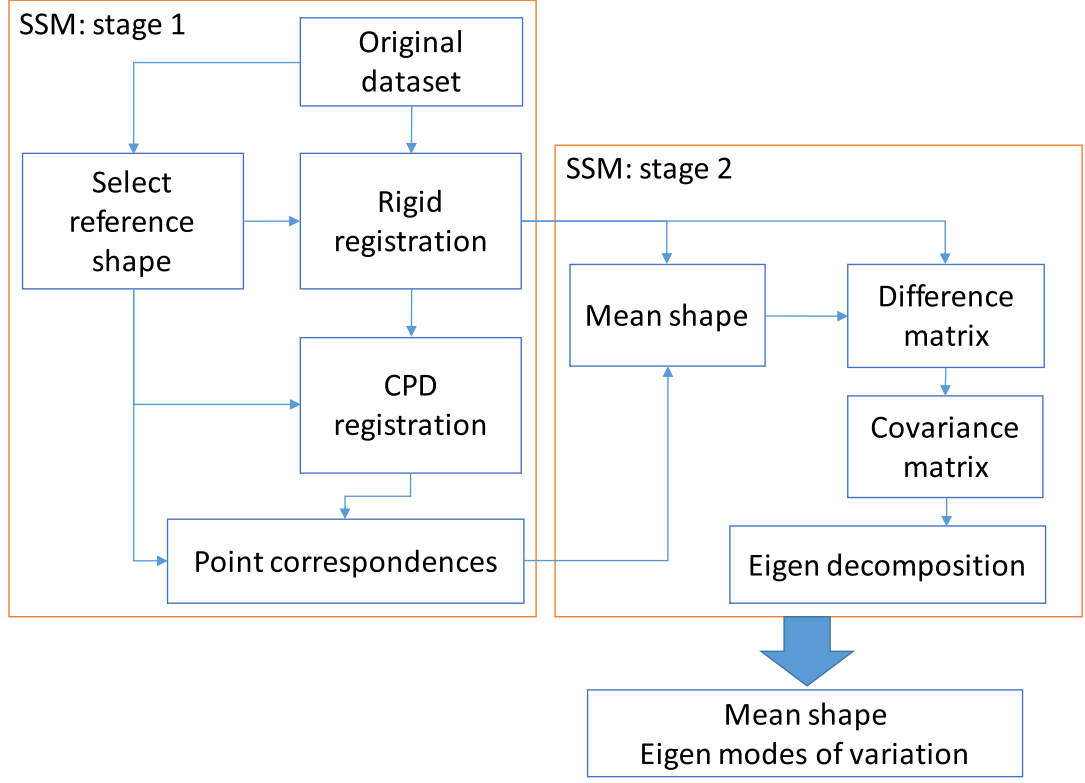

FIGURE 3 Two-stage procedure to compute the statistical shape model. In stage 1 , the point correspondences among the surfaces across the dataset are determined. In stage 2, mean shape and covariance matrix are computed 


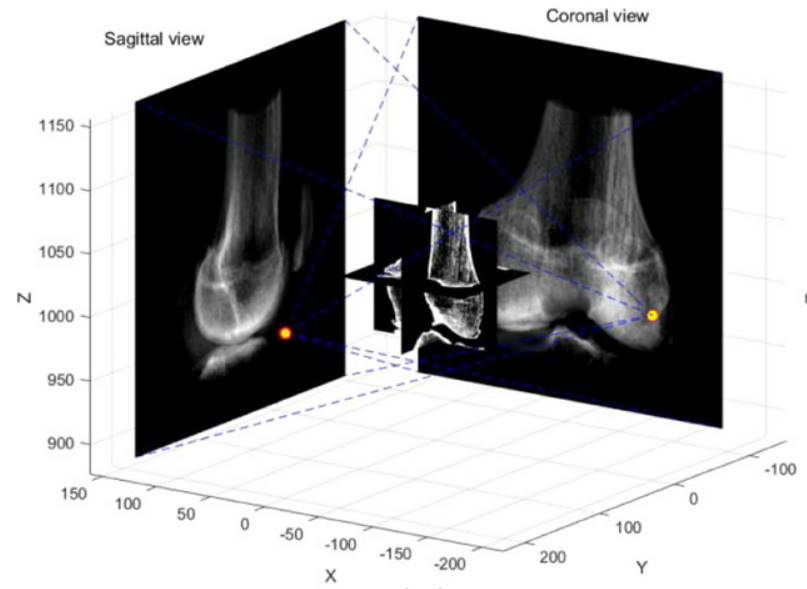

(A)

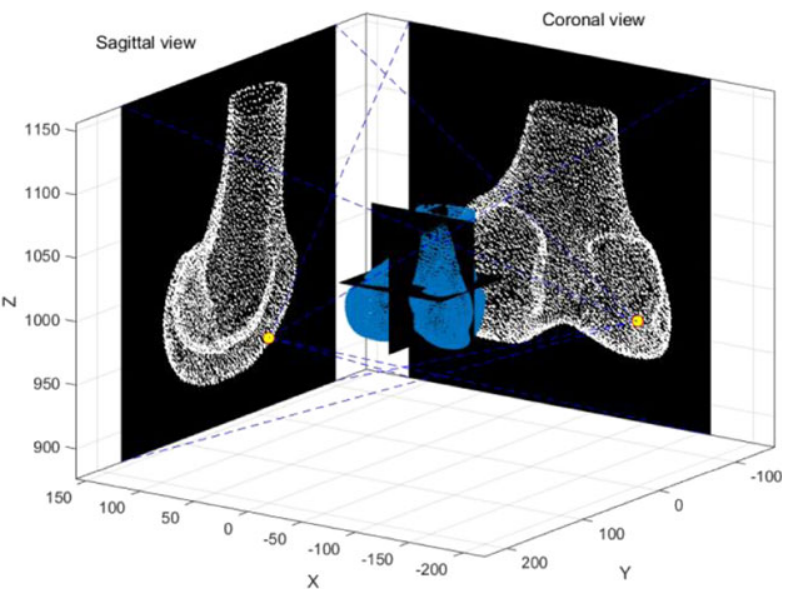

(B)

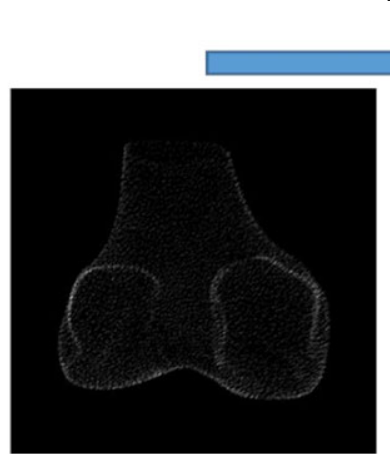

Original DRR

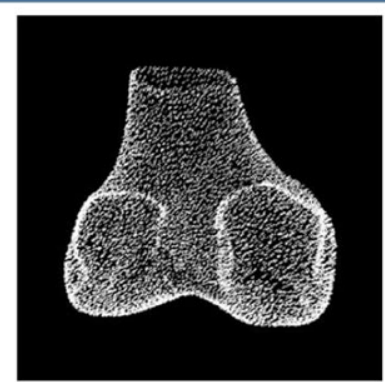

Intensity-rescaling

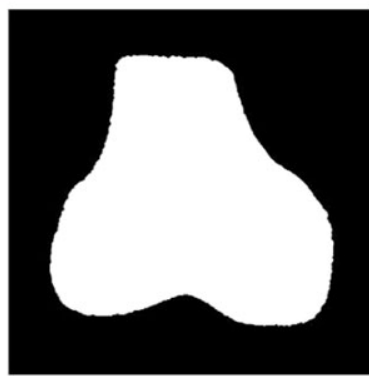

Gabor filter

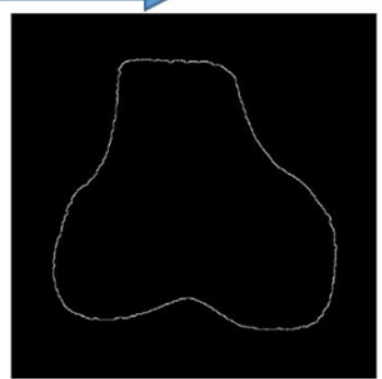

Canny edge detection

(C)

FIGURE 4 Two orthogonal DRR (sagittal and coronal projection) of the patient CT volume (A) and the CT-equivalent volume obtained from the morphed surface (B). Yellow spots indicates source location. The source to detector distance, detector size and DRR resolution were $450 \mathrm{~mm}$, $280 \mathrm{~mm}$ and $512 \times 512$, respectively. DRR image processing workflow (C)

the images were manually elaborated to remove both the patella and the tibia. Then they underwent the same processing as the sDRR to extract the contours. Once the contours on corresponding DRR were available, elastic registration ${ }^{27}$ allowed determining the $2 \mathrm{D}$ correspondences. The fitness quantity to be minimized in the reconstruction procedure was computed as:

$$
f=\frac{S_{x}}{N_{x}} \frac{\sum_{i}^{P} \sqrt{e_{i}^{2}}}{P} \quad \text { with } e_{i}=\sqrt{\sum_{j}^{Q_{i}}\left(p_{i, j}^{s}-p_{i, j}^{v}\right)^{2}}
$$

where $P, Q_{i}, p_{i, j}^{s}, p_{i, j}^{v}, S_{x}, N_{x}$ are the number of virtual $\mathrm{X}$-ray projections, the number of corresponding points in the two ith SDRR and VDRR, the jth image point in the ith sDRR, the jth image point in the ith vDRR, the detector width $(\mathrm{mm})$ and the number of pixels of the detector width, respectively.

\section{3 | Reconstruction procedure}

The reconstruction procedure was based on maximizing the $2 \mathrm{D}$ contour matching between SDRR and vDRR.

An iterative optimization algorithm, based on evolution strategy with covariance matrix adaptation (ES-CMA) was implemented. ${ }^{31,32}$ In this framework, the target variables (parameter set) encompassed an affine transform and the morphing $\left\{a_{j}\right\}$ parameters. The affine transform accounted for a global scale $\gamma$, to cope with scale difference between the SSM and the patient knee size, three translations and three orientations. The optimization evaluated a-posteriori the 2D matching quality (Equation (6)) provided by the actual parameter set (Figure 5).

At each iteration step, a population of $K$ parameter sets was generated throughout a mutation function applied to a parameter set prototype, namely the father of the population. For each parameter set in the population, the procedure generated from the mean shape the corresponding morphed surface, computed the DRR, performed image processing and calculated the fitness (Equation (6)). After computing the fitness of the all parameter sets in the current population, the procedure sorted the parameter sets in agreement to decreasing fitness. A subset of $\mu$ parameter sets was used to compute the father at the next iteration step by the recombination function. Then, the mutation function was updated according to the subset. This optimization strategy is called ES-CMA with rank- $\mu$ update. $^{33}$ It was shown that this method equals natural gradient descent with the advantage that the learning rate is intrinsically updated according to the local fitness function landscape. The optimization automatically stopped when the step size of the mutation function was below a predefined convergence threshold, which was set to 0.001 . 


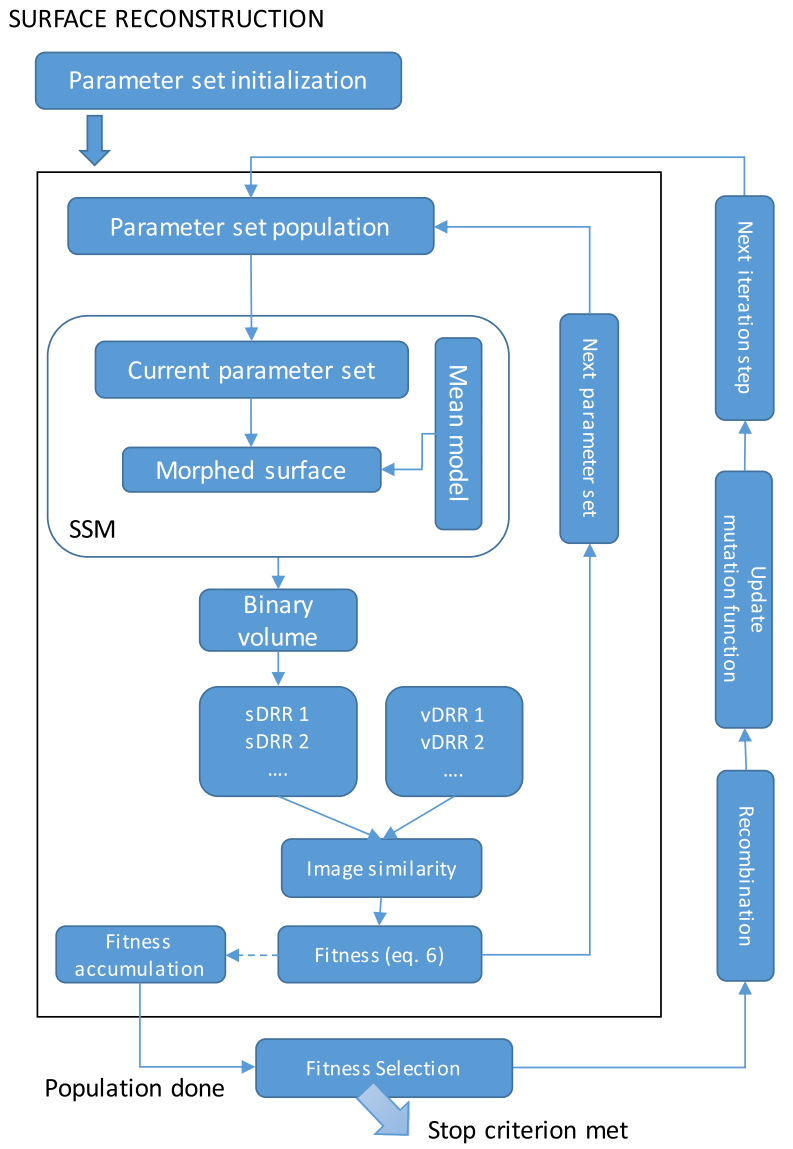

FIGURE 5 Iterative reconstruction procedure based on evolution strategy

\section{3 | EXPERIMENTAL TESTS AND RESULTS}

The reconstruction quality was quantitatively evaluated by the root mean squared error $e$ and Hausdorff distance $h$ (median, lower and upper quartile) between the morphed surface and the corresponding nominal surface. Qualitatively, color-based error maps were superimposed to the nominal surface to highlight local errors. The validation of the reconstruction method was performed with six tests where the source to detector distance, detector size and DRR resolution were set to $450 \mathrm{~mm}, 280 \mathrm{~mm}$ and $512 \times 512$, respectively.

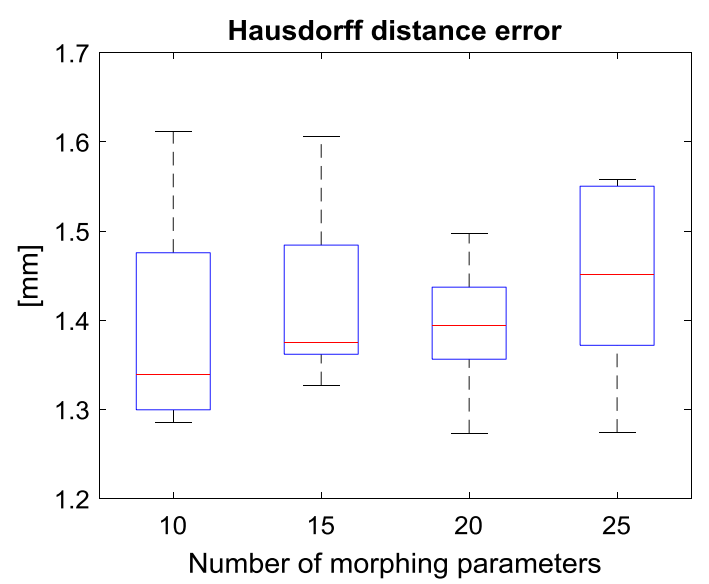

\section{1 | Test 1: morphing parameter number}

In the first test, five shapes, randomly selected in the testing dataset, were reconstructed using alternatively 10, 15, 20 and 25, upon the 80 available, morphing parameters, corresponding to $75 \%, 81 \%, 86 \%$ and $90 \%$ of explained variance (Equation (3)), respectively. Three DRR, being all orthogonal to the axial direction, namely one sagittal $\left(0^{\circ}\right)$, one coronal $\left(90^{\circ}\right)$ and one oriented at $45^{\circ}$ with respect to the sagittal view were used. Results of the median values of the distributions were statistically compared using the Kruskall-Wallis ANOVA equivalent statistical test with post-hoc comparison $(P=0.01)$. Reconstruction results showed a range of $h$ and $e$ median values lower than 1.50 and $0.80 \mathrm{~mm}$, respectively (Figure 6). The median errors slightly increased as the number of parameters increased but no statistical difference $(P>0.8)$ was found. This led to consider that 10 morphing parameters are sufficient to ensure high reconstruction accuracy.

\section{2 | Test 2: number of DRR}

In the second test, all the 20 test surfaces were reconstructed using 10 morphing parameters and the accuracy was evaluated as a function of the number of DRRs, encompassing two, three, four and five projections (Table 1). The three error distributions were statistically compared using Kruskal-Wallis with post-hoc comparison $(P=0.01)$.

The $h$ and $e$ median values were lower than 1.8 and $1.00 \mathrm{~mm}$, respectively (Figure 7). As expected, increasing the number of DRR improved the reconstruction quality. Statistical difference $\left(P<10^{-5}\right)$ was found in the 2 vs 3,2 vs 4 and 2 vs 5 DRR comparisons. This led to consider that 3 DRR provide enough shape information to ensure

TABLE 1 DRR setup in test \#1. The source-detector orientation of $0^{\circ}$, $45^{\circ}, 90^{\circ}, 135^{\circ}$ and $180^{\circ}$ were around the axial direction with respect the sagittal view

\section{Orientation}

\begin{tabular}{lccccc}
\hline DRR\# & Sagittal $\left(0^{\circ}\right)$ & Coronal $\left(90^{\circ}\right)$ & $45^{\circ}$ & $135^{\circ}$ & $180^{\circ}$ \\
\hline 2 & $\times$ & $\times$ & & & \\
3 & $\times$ & $\times$ & $\times$ & & \\
4 & $\times$ & $\times$ & $\times$ & $\times$ & \\
5 & $\times$ & $\times$ & $\times$ & $\times$ & $\times$ \\
\hline
\end{tabular}

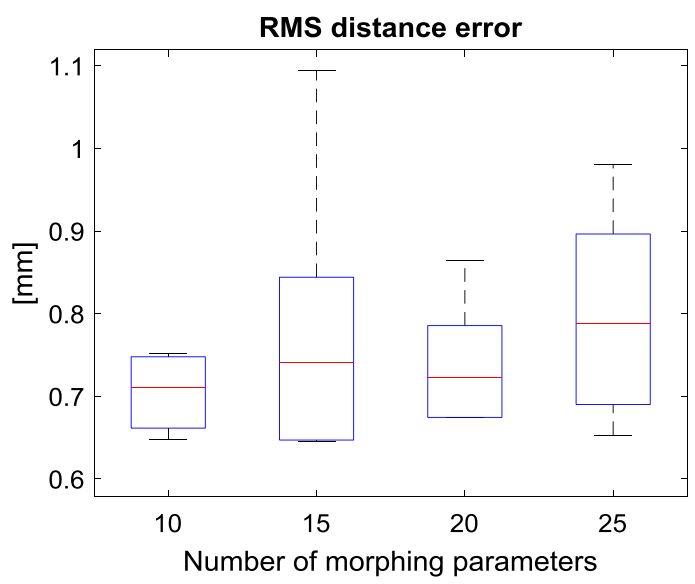

FIGURE 6 Reconstruction distance error as a function of the number of morphing parameters. The median values (red lines), along with the 25 and 75 percentiles (lower and upper box boundaries) were reported for both the Hausdorff and RMS error distributions 

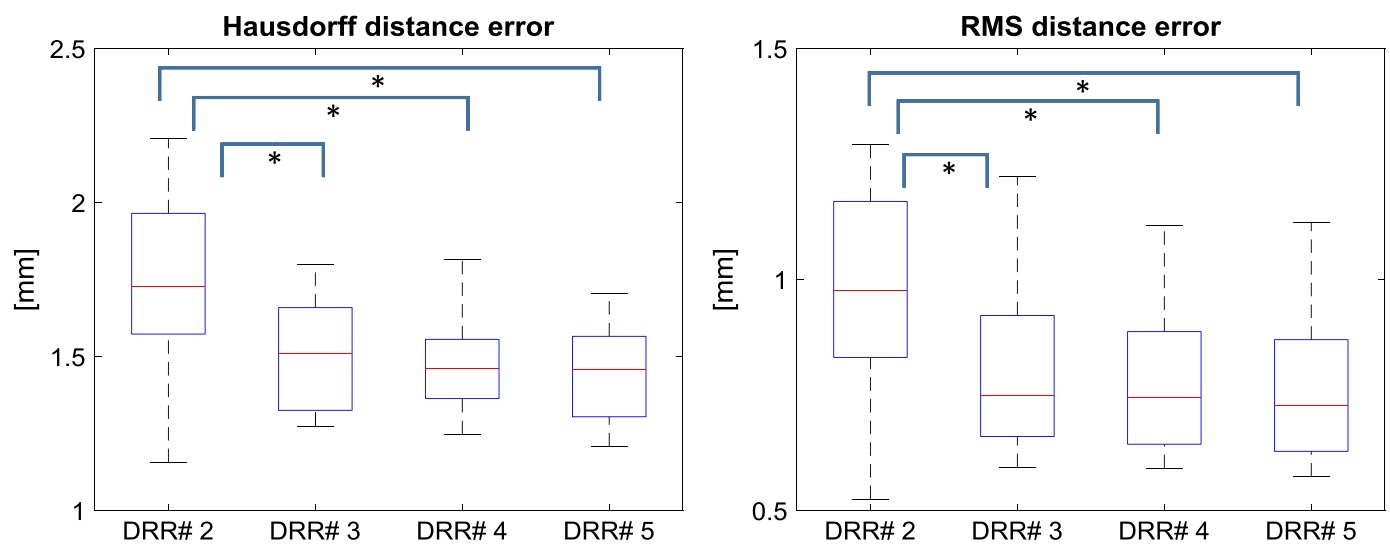

FIGURE 7 Reconstruction errors (test \#2) computed over the testing dataset of 20 shapes using 10 morphing parameters. Statistical difference $(P<10-5)$ was found when comparing from 2 to 3,2 to 4 and 2 to 5 DRR. No difference was detected in the other comparisons. The median values (red lines), along with the 25 and 75 percentiles (lower and upper box boundaries) were reported for both the Hausdorff and RMS error distributions

high accuracy. Qualitatively, the increase of the number of DRR reflected on a better reconstruction at the facets of the trochlear and posterior condylar regions (Figure 8). Also, epicondylar processes (see Figure 9 - patient 98) were better reproduced when increasing the number of DRR.

\section{3 | Test 3: reconstruction errors on the condylar regions}

In the third test, the local errors at distal condylar level, namely anterior medial, anterior lateral, posterior medial and posterior lateral portions, were computed for 2, 3, 4 and 5 DRRs reconstruction. An automated procedure, previously described in the literature, ${ }^{34}$ was applied to extract the four condylar portions. Statistical differences among the reconstruction errors at the four regions were evaluated using Kruskal-Wallis test $(P=0.01)$ for the equality of median values and Levene test $(P=0.01)$ for equality of the dispersions. Local reconstruction errors computed at the distal lateral posterior (LLP), distal medial posterior (MLP), distal lateral anterior (LLA), distal medial anterior (MLA) as a function of the number of DRR were again dependent on the number of the used DRR (Figure 9). No statistical differences for the median values of the error distributions among the four anatomical regions (DRR2: $P=0.32$; DRR\#3: $P=0.68$; DRR\#4: $P=0.19$; DRR\#5: $P=0.62$ ) were detected. Similarly, the four corresponding dispersions were not statistically different in between, being this valid in each of the four DRR setups.

\section{4 | Test 4: effects of PSI contact area error on surgical planning}

In order to evaluate the morphologic fitting of the PSI to the reconstructed surface and the expected deviation of the planned surgical cuts, we evaluated the reconstruction errors at the four contact areas, namely anterior lateral, anterior medial, distal medial and distal lateral, traditionally used in MyKnee surgical planning (Figure 10, upper panel). The boundary of each area was sampled with four technical points (basically at the vertices of the area), which were used to compute the distance error to the corresponding points, determined by minimal distance, on the reconstructed surface. Without lack of generality, we considered the impact of contact area errors on the deviation of the distal cut direction. Contact area errors were hypothesized to produce a pure $3 D$ rotation of the distal cut direction, which was analyzed in the frontal and axial planes. The rotation in the frontal plane was estimated over the eight technical points on the distal medial and distal lateral contact areas. The rotation in the sagittal plane was estimated over the eight technical points on the anterior lateral and anterior medial contact areas. Each rotation was computed throughout a constrained estimation of the transform between the eight points on the planning surface and the corresponding eight points on the reconstructed surface. The overall test was performed across the 20 test patients using 10 morphing parameters and alternatively two, three and four DRRs (see Table 1). In distance error results were averaged across the four points in each areas and then averaged across the 20 patients. As expected, the maximum residual error was obtained when using 2 DRR only (Table 2).

The errors on the contact areas were approximately similar to the errors reported for the condylar regions (see Figure 9). The effect of the frontal contact area errors on the distal cut direction was basically dependent again on the number of used DRRs (Figure 10, lower panel), with a maximum median error lower than $2^{\circ}$ and $0.5^{\circ}$ on the sagittal and frontal plane, respectively. Interestingly, all the 20 cases showed deviations of the distal cut direction in the frontal plane lower than $3^{\circ}$, which can be considered clinically acceptable. However, the greater deviations in the sagittal plane should be carefully regarded as the corresponding malalignment of the mechanical axis of the femur modifies the tibio-femoral extension gap, which in turn affects the patellofemoral joint kinematics and ligament balancing.,

\section{5 | Test 5: effect of off-the-axial-plane DRR}

The fifth test involved the use of one off-the-axial-plane projection, with increasing angle $\left(0^{\circ}, 10^{\circ}, 20^{\circ}, 30^{\circ}, 40^{\circ}, 50^{\circ}\right)$, in addition to the coronal and sagittal views, oriented at $45^{\circ}$ in the axial plane. Ten patients were selected randomly in the testing dataset and the reconstruction results were compared with the reconstruction of the same patients obtained using three DRR (see Table 1). The reconstruction error 

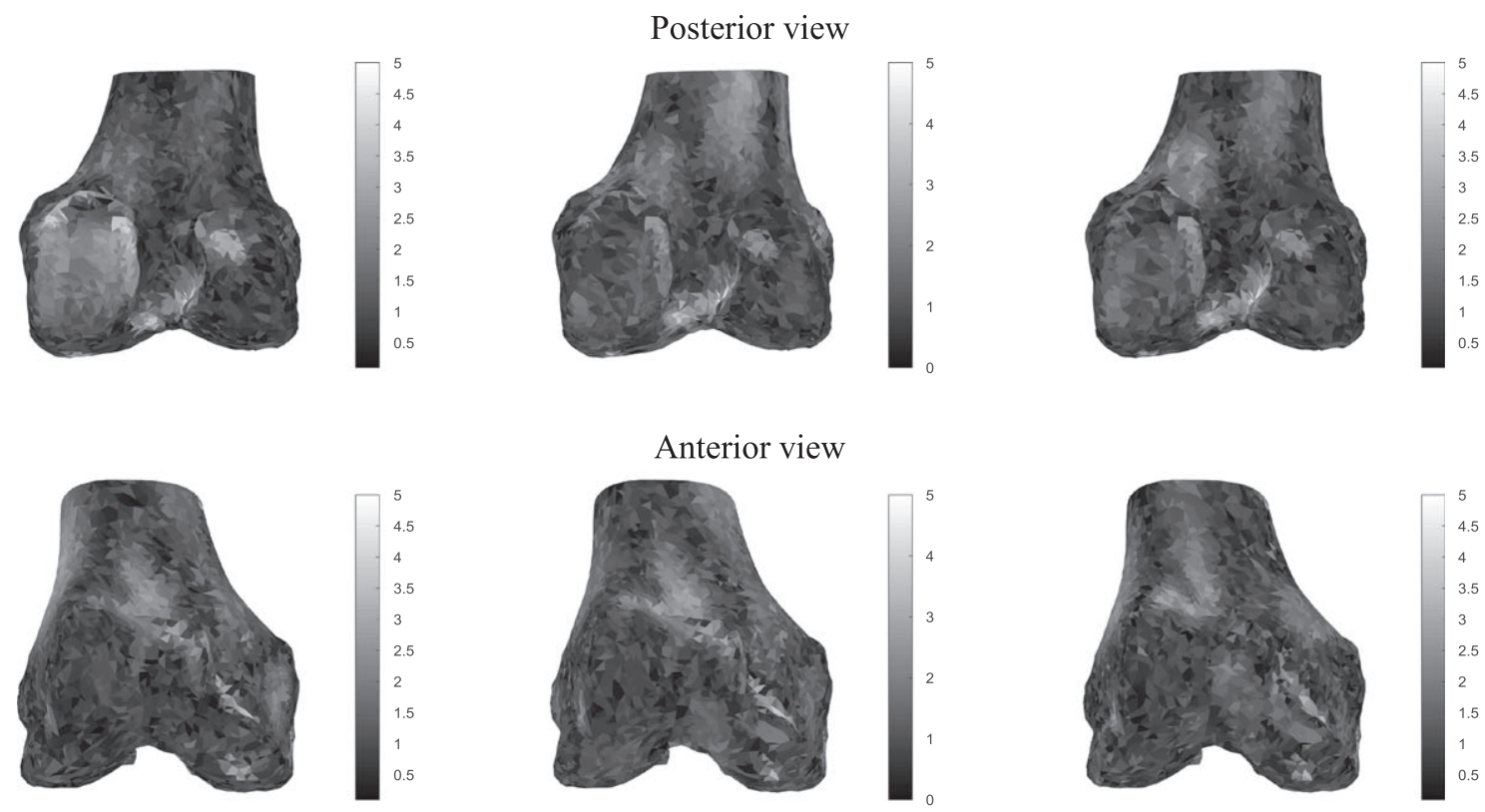

$\mathrm{P}$.
81

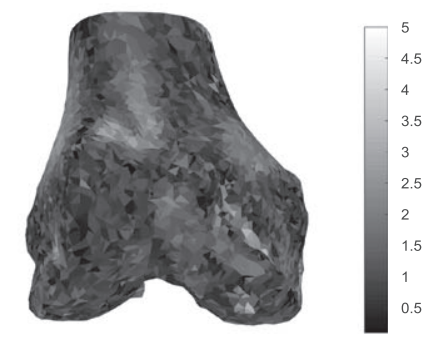

Sagittal view
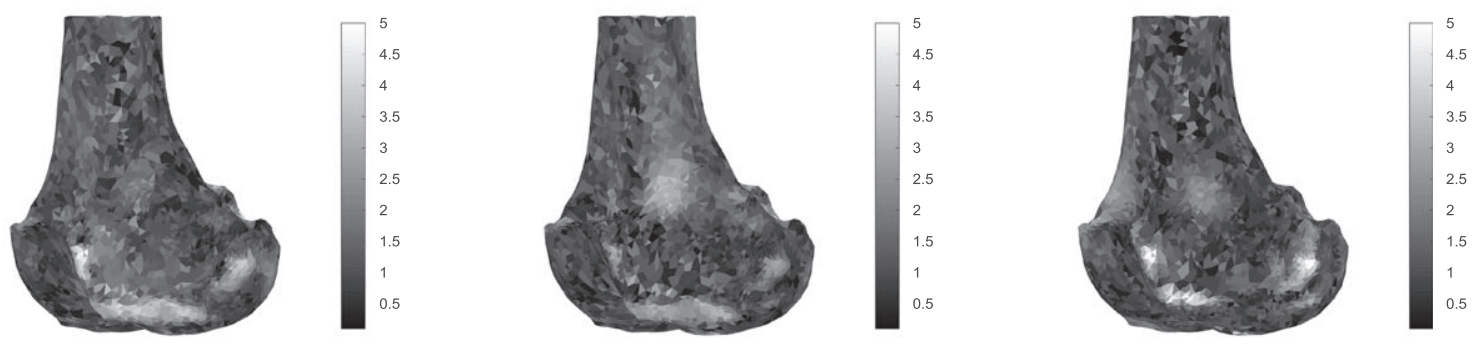

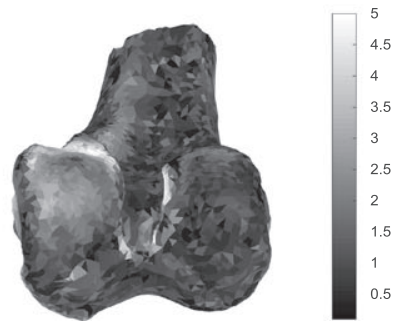

DRR\# 2
Posterior view

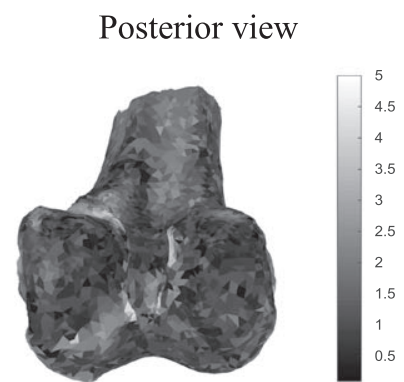

DRR\# 3

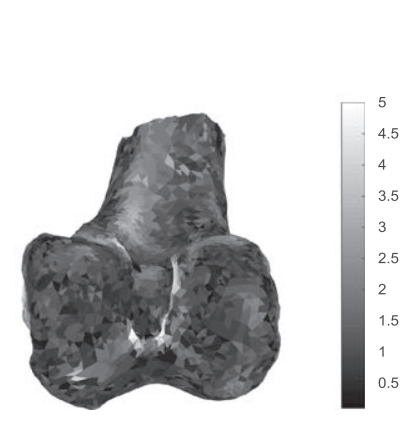

DRR\# 4

FIGURE 8 Color-mapped reconstruction errors $(\mathrm{mm})$ for patients 81 and 98 in test \#2. Main differences appears on the trochlear facets and the condylar regions

distributions for 10 patients, randomly extracted in the test dataset, as a function of the off-the-axial-plane DRR angle, showed interestingly that lower median errors were found between 20 and 30 degrees and were larger when both decreasing and increasing the angle of the third DRR (Figure 11).

\section{6 | Test 6: comparison with SSM built using non- pathological distal femurs}

The sixth test envisaged the comparison between the reconstructions performed through the SSM built on the 80 pathological shapes and a different SSM built on 20 distal right femur surfaces, reconstructed from CT scans of cadavers with a mean age of 77 years (61-95). ${ }^{34}$ No femoral morphologic anomalies were described in this latter dataset. Basically, the comparison between the two SSMs, namely one built with pathologic femurs and one built with normal femurs, was to verify whether osteophytes can be extrapolated by the SSM built with normal anatomies.

Distal femur surfaces were obtained through manual segmentation, performed by expert orthopedic radiologists using Mimics software (Materialise NV, Leuven, Belgium), and resampled to 8000 vertices. For the SSM computation, some 6000 correspondences were found across the 20 training femur shapes. For the reconstruction step, the DRR setup encompassed three projections (cf. Table 1) and 10 

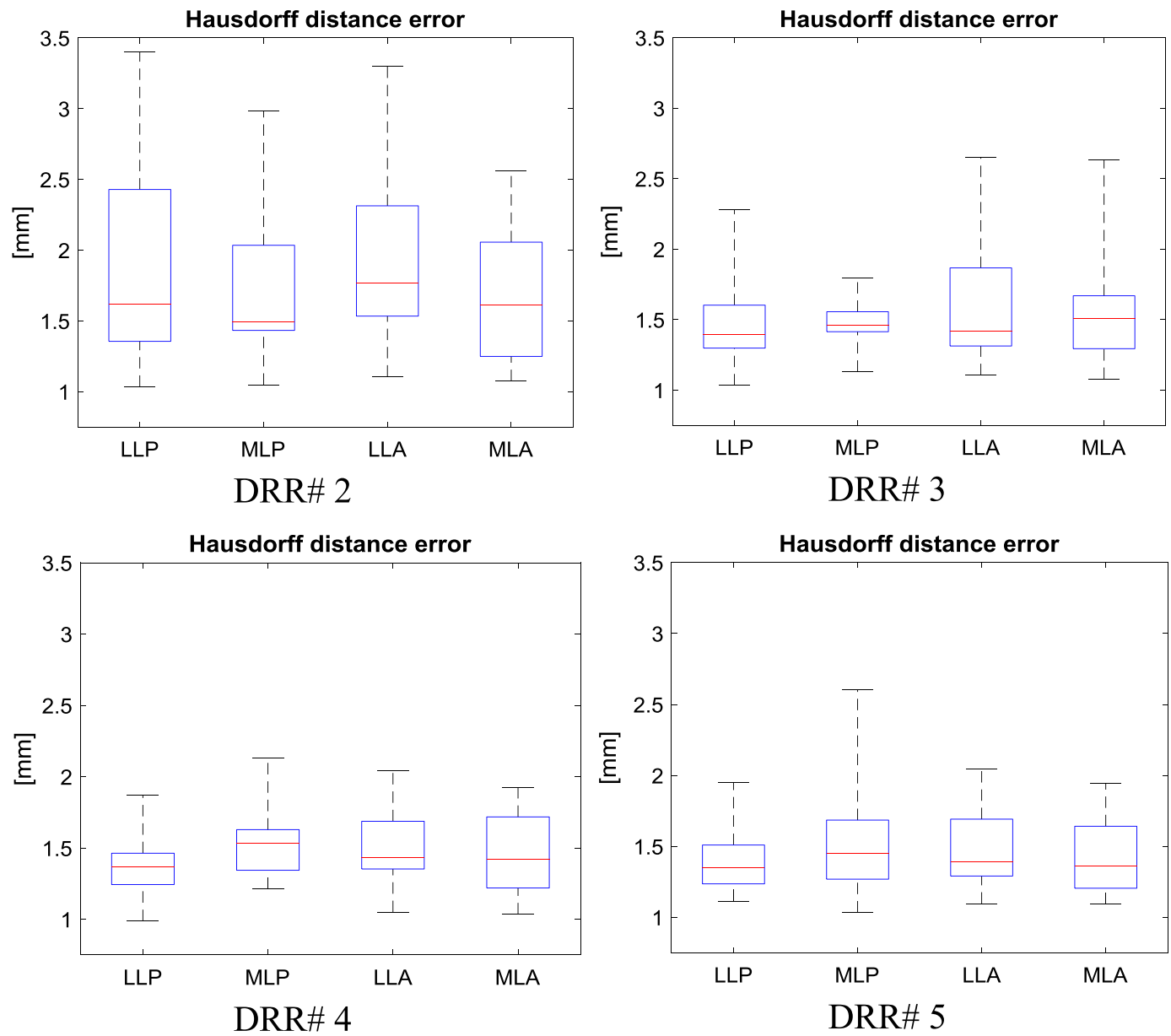

FIGURE 9 Local reconstruction errors (test \#3) computed at the distal lateral posterior (LLP), distal medial posterior (MLP), distal lateral anterior (LLA), distal medial anterior (MLA) portions of the two condyles as a function of the number of DRR. The median values (red lines), along with the 25 and 75 percentiles (lower and upper box boundaries) were reported for both the Hausdorff and RMS error distributions

morphing parameters were chosen, featuring about $88 \%$ of the explained variance. Again, Kruskal-Wallis test was used $(P=0.01)$.

The two distributions of the reconstruction errors on the test dataset (20 patients), with median values of 1.50 and $2.03 \mathrm{~mm}$, respectively, were statistically different $(P<0.0001)$ (Figure 12). The distribution of the reconstruction errors by SSM normal showed also a higher dispersion. As an example, we depicted the color-mapped reconstruction errors for the patient 81 using the SSM pathologic (Figure 13 lower left panel) and SSM normal (Figure 13 lower right panel). As a result, the reconstruction based on the SSM normal was unable to reproduce correctly the medial condylar ridge in the distal direction.

\section{4 | DISCUSSION AND CONCLUSIONS}

\section{1 | Main findings}

The main findings of this paper can be summarized as follows. Even with a high number of variation modes in the SSM, 10 morphing parameters, that explained $75 \%$ of the variations in the training dataset, were sufficient to reconstruct the distal femur surface with accuracy depending only on the DRR setup. Three DRR images (e.g. sagittal, coronal, $45^{\circ}$, all orthogonal to the axial direction) provided an accuracy (Hausdorff distance error) of the reconstruction in the range of $1.5 \mathrm{~mm}$, being significantly different from the accuracy obtained using two DRR only $(2.00 \mathrm{~mm})$. Greater reconstruction errors were spatially located at the trochlear and condylar regions that are basically the parts of the distal femur undergoing most of morphologic anomalies due to knee arthrosis. Consistent with earlier literature, adding more DRR improved the local accuracy (see Figure 8). However, no reconstruction differences were found among the anterior/posterior and medial/lateral portions of the condyles (see Figure 9). Low influence of the PSI contact area errors on the distal cut direction in both sagittal and frontal planes was found (see Figure 10). Improvements of the reconstruction accuracy were obtained by using off-the-axial-plane images (see Figure 11) suggesting that displaced DRRs are more appropriate in a realistic setup. The SSM built on normal femurs led to worse reconstruction accuracy especially at condylar level (see Figure 12), thus confirming that the reproduction of specific morphologic variations is more effective if the training dataset embeds such variations sufficiently. Pathological conditions of the distal femur do not appear simply as smooth deformations of the condyle surfaces but rather unpredictable bony growth localized all over the condylar development even involving distributed spurs on the anterior part of the trochlear region. This suggests that the inclusion of many cases 

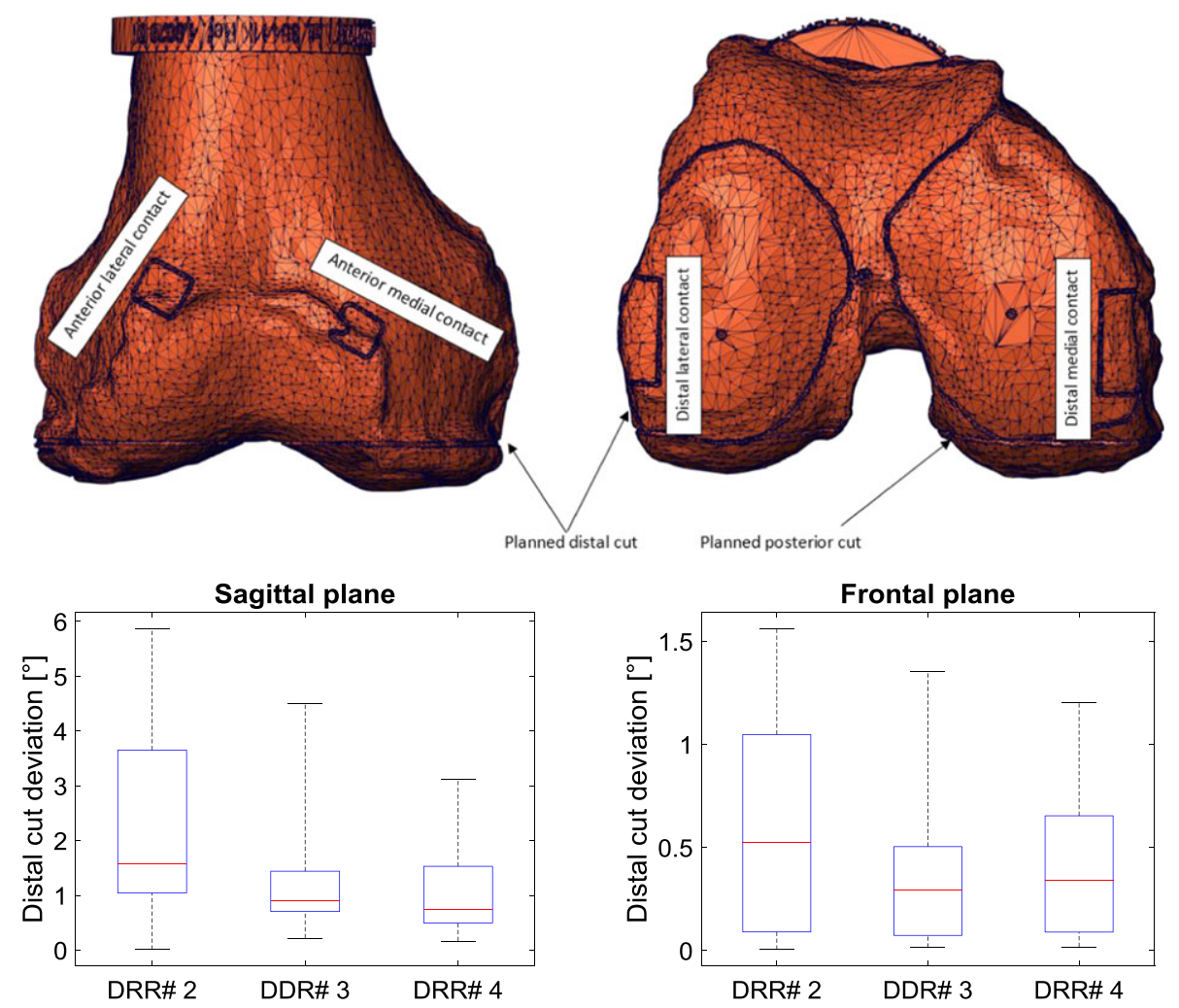

FIGURE 10 Frontal and axial views of the femoral planning of the cuts. We highlighted the contact areas of the PSI femoral component (upper panel). Variation of the orientation of the distal cut direction on the sagittal and frontal planes as a function of the number of DRRs. No statistical difference (Kruskall-Wallis non-parametric test) among the three distributions in both planes was detected $(P>0.1)$. The median values (red lines), along with the 25 and 75 percentiles (lower and upper box boundaries) were reported for both the Hausdorff and RMS error distributions (lower panel)

TABLE 2 Accuracy (mean error \pm standard deviation) results on the PSI contact areas

\begin{tabular}{lcccc} 
& Anterior lateral $(\mathrm{mm})$ & Anterior medial $(\mathrm{mm})$ & Distal lateral $(\mathrm{mm})$ & Distal medial $(\mathrm{mm})$ \\
\hline 2 DRR & $1.47(0.37)$ & $1.70(0.52)$ & $1.21(0.21)$ & $1.69(0.48)$ \\
3 DRR & $1.31(0.35)$ & $1.73(0.60)$ & $1.14(0.29)$ & $1.51(0.38)$ \\
4 DRR & $1.33(0.35)$ & $1.68(0.45)$ & $1.14(0.30)$ & $1.41(0.31)$ \\
\hline
\end{tabular}
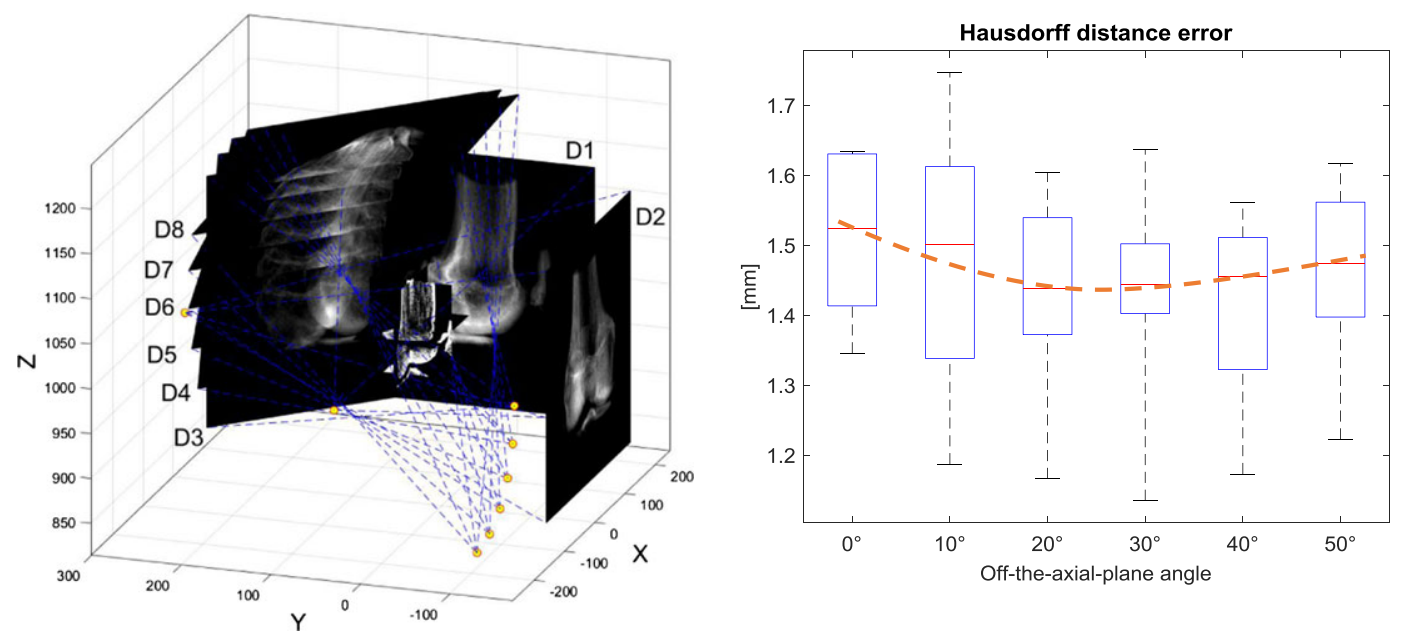

FIGURE 11 (Right panel) DRR setup: one sagittal, one coronal and off-the-axial-plane DRR. Detectors D3, D4, D5, D6, D7, D8 corresponds to angular displacements of $0^{\circ}, 10^{\circ}, 20^{\circ}, 30^{\circ}, 40$ and $50^{\circ}$, respectively. (Left panel) Error distributions of the reconstruction of 10 patients in the test dataset. A spline curve was fit on the median values 

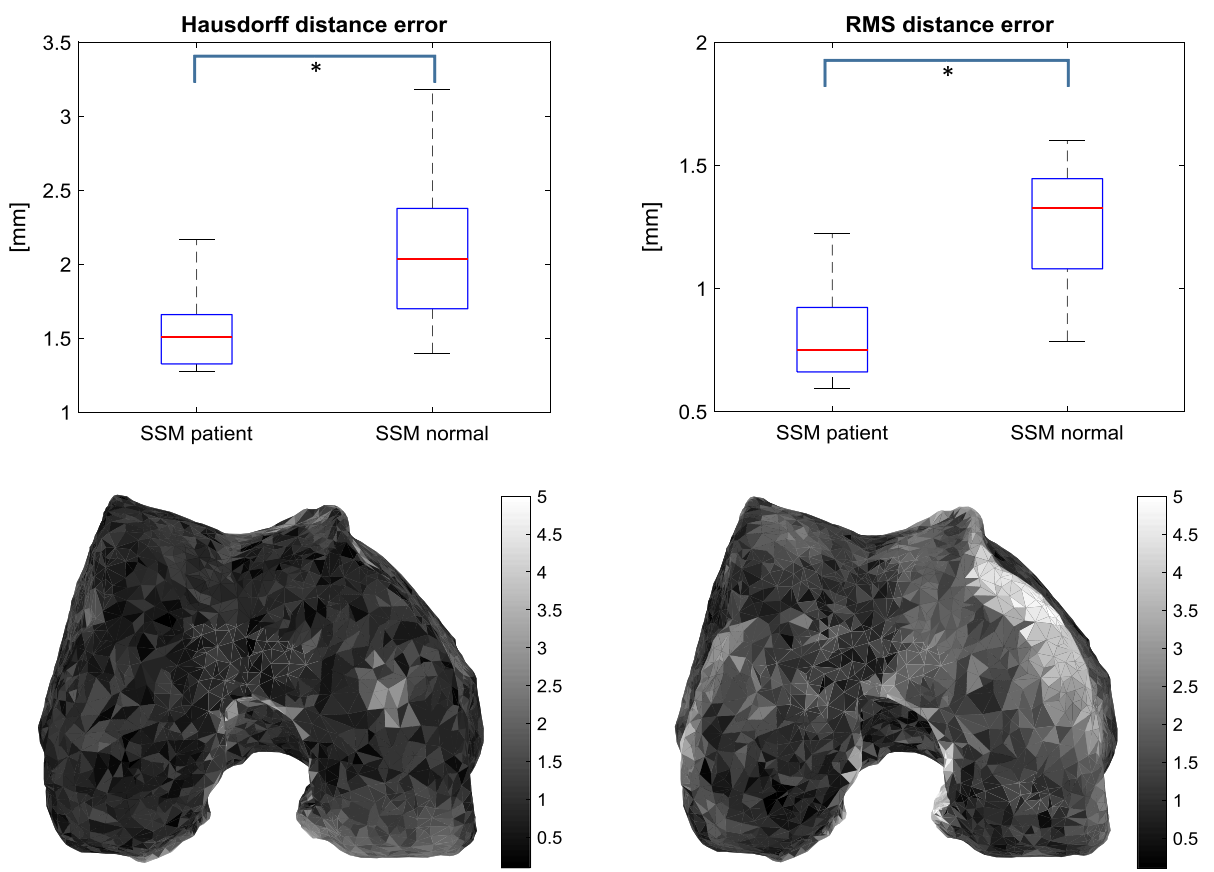

FIGURE 12 (Upper panel) Error distributions of the test dataset (20 patients) reconstructed using the SSM built on 80 pathologic (Hausdorff median error: $1.50 \mathrm{~mm}$; RMS median error: $0.74 \mathrm{~mm}$ ) and the SSM built on 20 normal femurs (Hausdorff median error: $2.03 \mathrm{~mm}$; RMS median error: $1.32 \mathrm{~mm}$ ) (DRR\# 3), respectively. Both distributions were statistically different $(P<0.0001 ; P<0.00001)$. The median values (red lines), along with the 25 and 75 percentiles (lower and upper box boundaries) were reported for both the Hausdorff and RMS error distributions. (Lower panel) Color-mapped reconstruction errors $(\mathrm{mm})$ for the patient 81 (axial view): SSM pathologic (left), SSM normal (right)
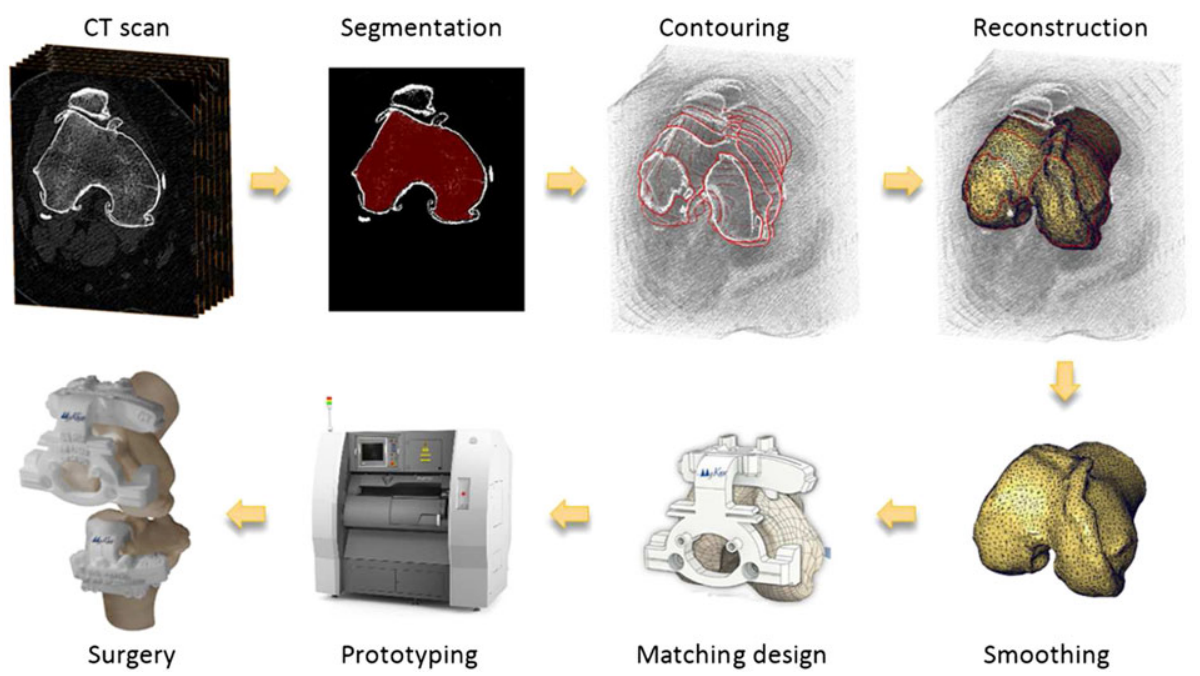

Smoothing

FIGURE 13 Technical workflow chart of the PSI

encompassing pathologic variations due to osteophytes is mandatory in the SSM training dataset. $^{25}$

\section{2 | Comparison with other SSM approaches}

SSMs were extensively studied for 2D/3D bone reconstruction. In, ${ }^{17}$ the authors proposed one SSM, built on non-pathologic proximal femurs, to reconstruct the proximal femur shape from multiple $X$-ray images. They reconstructed 23 proximal (five pathologic) and 18 (non-pathologic) femurs with a RMSE of about $0.75 \mathrm{~mm}$, with no statistical difference between the two sets. The low number of pathologic femurs prevented however to generalize the extent of such a result. The other limit of the study was that the pathologic variations were not described. In the present paper, we use specific pathological knees affected by osteophytes at condylar level.

$\operatorname{In}{ }^{18}$ the authors proposed a single frontal X-ray image to reconstruct pelvic bone for estimating postoperative cup orientation. While they reported good agreement with the angular orientation obtained from $\mathrm{CT}$, they did not evaluate the surface reconstruction accuracy. $\ln ^{19}$ the authors used 40 normal distal femur shapes from adults, reconstructed from MRI scans, to build a SSM. The RMSE using two images was in the range of $0.65 \mathrm{~mm}$ in agreement with our results $(0.75 \mathrm{~mm}$ ) obtained using 80 pathological shapes to build the SSM. Two main drawbacks limited the span of such work: (1) no pathologic anomalies were taken into account in the SSM; (2) the validation was performed using the same shapes adopted to build the SSM. 
$\mathrm{In}^{35}$ the authors evaluated the possibility of investigating in vivo knee kinematics by means of a dual-fluoroscopic imaging system using a SSM was built upon 153 healthy subjects. The reconstruction errors of the femur surface were in the range of about $1.20 \mathrm{~mm}$, again in agreement with our results. However, the disregard of pathologic shapes and the small testing cohort (three subjects) did not demonstrate the chance of using SSM for reconstructing with accuracy morphologic anomalies.

\section{3 | Clinical impact}

For the femoral resection component of MyKnee PSI, the coupling mainly depends on the reconstruction accuracy of the frontal and distal aspects of the distal femur, whereas for the tibia, the matching is affected by the coupling to the frontal aspect of the tibial plateau. Nominally, the final coupling tolerance should reflect the combination of uncertainty in the scanning resolution, the image segmentation accuracy, the surface reconstruction, the surface smoothing, the digital representation of the surface in the manufacturing system and finally the manufacturing precision (Figure 13). While it is not straightforward to quantify exactly the error chain in each step, we provide a reasonable guess of the final matching error between the PSI and the true patient anatomy. The scanning resolution, namely the voxel size, which is typically $0.5 \times 0.5 \times 0.5 \mathrm{~mm}$ for both planning CT and MRI, is the first source of uncertainty. When carefully performed, the segmentation quality, which however depends on the image modality (in the MRI the bone-soft tissue boundary is less contrasted than that in the CT) and on the operator performing the task, introduces a further uncertainty that can be assumed at sub-voxel size level. It is well known however that the high quality segmentation process is very time consuming. The surface reconstruction quality, which depends on slicing thickness (e.g. $0.5 \mathrm{~mm}$ ), and the surface smoothing further increase the uncertainty. Assuming that the virtual matching in the PSI design is perfect, no additional errors should be considered at this stage. Contrary, the prototyping process can decrease further the matching accuracy due to the internal representation of the surface data and the manufacturing precision. For instance, modern 3D printers ensure a precision of $0.2 \mathrm{~mm}$. In conclusion, it is realistic to assume that, in the best condition, the uncertainty of the coupling is in the range of $1 \mathrm{~mm}$. It was reported that such uncertainty can induce rotational differences in the coronal and sagittal planes, between the planned and intra-operative alignment by $\mathrm{PSI}$, in the range of about $2^{\circ} .{ }^{36}$ Using three DRRs, we showed that it is possible to attain a reconstruction accuracy in terms of Hausdorff distance lower than $1.5 \mathrm{~mm}$ and a RMSE lower than $0.75 \mathrm{~mm}$, with respect to the nominal distal femur surface reconstructed from CT scans, result that can be improved by adding more DRRs, thus granting sufficient accuracy for PSI matching. We reported that such reconstruction errors can produce reasonably deviations of the direction of the distal cut in both frontal and sagittal planes similar to those ones reported in ${ }^{36}$ and, ${ }^{37}$ thus being in the range of tolerable surgical errors. Therefore, using SSM and X-ray images can cope in principle with PSI accuracy requirements. The introduction in the orthopedic practice of mobile C-Arm X-ray systems permits the acquisition of a number of calibrated $X$-ray images sensibly ensuring the reduction of the radiation exposure to patients
(0.001 mSv per X-ray), compared with using CT images $(0.16 \mathrm{mSv}) .{ }^{12,26}$ We have, however, to remark that the role of the PSI surgical technique has been topic of many debates with no general consensus about the accuracy and reliability for a large scale surgery..$^{6-9}$ While PSI cannot be regarded as the gold standard in total knee replacement, advanced osteoarthritis conditions can be surgically addressed through such a technique, especially in conditions of bone deformity, which can prevent the use of intra-medullar bars. ${ }^{10,11}$

\subsection{Implementation issues}

The SSM training and the reconstruction process were implemented in the Matlab-2016a suite (Mathworks, Natick, MA) and were both completely automatic. The volume binary computation, ray-casting projection and deformable registration were $\mathrm{C}$-compiled in executable Matlab mex files. In the reconstruction, all the parameters (affine transform + morphing variables) were estimated in bundle making the computational cost quite high. Due to the genetic-based iterative optimization, the reconstruction with three DRR took about $45 \mathrm{~min}$ on an $17 \mathrm{CPU}$ Intel $2.6 \mathrm{GHz}$ clock speed with $8 \mathrm{~GB}$ RAM, making mandatory code optimization for future developments.

\section{5 | Final remarks}

In summary, this study evaluated the feasibility of using SSM to reconstruct the 3D surface the distal femur for PSI application in total knee replacement. The RMSE of the reconstruction was lower than $0.75 \mathrm{~mm}$ when using three DRRs with a corresponding deviation of the direction of the planned distal cut lower than $2^{\circ}$. The $2 D / 3 D$ reconstruction do not require $\mathrm{CT}$ or $\mathrm{MR}$ imaging so avoiding processing volumetric scans that are known as being a challenge in clinical image analysis. While we did not use real calibrated X-ray images, realistic simulations demonstrated the feasibility of the method. We acknowledge that the in the real case, the geometry of the X-ray image acquisition needs to be known requiring calibration using fiducials. However, the on-going introduction of $\mathrm{C}$-arm radiographic systems in orthopedics would facilitate this stage. As a limitation of the present study, we disregarded gender differences, which were disclosed in previous studies, ${ }^{38}$ and did not consider tibia shapes, which are fundamental in PSI surgery. Future studies will address both these limitations.

\section{ACKNOWLEDGEMENTS}

We would like to thank Medacta International SA for providing patient data.

\section{CONFLICT OF INTEREST}

No party having a direct interest in the results of the research supporting this article has or will confer a benefit on the author(s) or on any organization with which the author(s) is/are associated.

\section{ETHICAL BACKGROUND}

The study involved retrospective anonymized image data provided by Medacta International SA. 


\section{REFERENCES}

1. Nam D, McArthur B, Cross M, et al. Patient-specific instrumentation in total knee arthroplasty: a review. J Knee Surg. 2012;25(03):213-220.

2. Ng VY, DeClaire JH, Berend KR, et al. Improved accuracy of alignment with patient-specific positioning guides compared with manual instrumentation in TKA. Clinic Orthopaed Related Res. 2011;470(1):99-107.

3. Renson L, Poilvache P, Van den Wyngaert H. Improved alignment and operating room efficiency with patient-specific instrumentation for TKA. Knee. 2014;21(6):1216-1220.

4. Mannan A, Smith TO. Favourable rotational alignment outcomes in PSI knee arthroplasty: a level 1 systematic review and meta-analysis. Knee. 2016;23(2):186-190.

5. Pietsch M, Djahani O, Hochegger M, et al. Patient-specific total knee arthroplasty: the importance of planning by the surgeon. Knee Surg Sports Traumatol Arthrosc. 2013;21(10):2220-2226.

6. Bali K, Walker P, Bruce W. Custom-fit total knee arthroplasty: our initial experience in 32 knees. J Arthroplasty. 2012;27(6):1149-1154.

7. Bugbee WD, Mizu-uchi H, Patil S, D'Lima D. Accuracy of implant placement utilizing customized patient instrumentation in total knee arthroplasty. Adv Orthoped. 2013;2013:1-6.

8. Nunley RM, Ellison BS, Ruh EL, et al. Are patient-specific cutting blocks cost-effective for total knee arthroplasty? Clinic Orthopaed Related Res. 2011;470(3):889-894.

9. Slover JD, Rubash HE, Malchau H, Bosco JA. Cost-effectiveness analysis of custom total knee cutting blocks. J Arthroplasty. 2012;27(2):180-185.

10. Seon J-K, Park H-W, Yoo S-H, Song E-K. Assessing the accuracy of patient-specific guides for total knee arthroplasty. Knee Surg Sports Traumatol Arthrosc. 2014;24(11):3678-3683.

11. Mattei L, Pellegrino $P$, Calò $M$, et al. Patient specific instrumentation in total knee arthroplasty: a state of the art. Annals Translation Med. 2016;4(7):126-126.

12. Biswas D, Bible JE, Bohan M, et al. Radiation exposure from Musculoskeletal computerized Tomographic scans. J Bone Joint Surg - Am Volume. 2009;91(8):1882-1889.

13. Cerveri P, Manzotti A, Confalonieri N, Baroni G. Automating the design of resection guides specific to patient anatomy in knee replacement surgery by enhanced 3D curvature and surface modeling of distal femur shape models. Comput Med Imag Graphics. 2014;38(8):664-674.

14. Cootes TF, Taylor CJ, Cooper DH, Graham J. Active shape models their training and application. Comput Vision Image Understanding. 1995;61(1):38-59.

15. Markelj $P$, Tomaževič $D$, Likar $B$, Pernuš F. A review of $3 D / 2 D$ registration methods for image-guided interventions. Med Image Anal. 2012;16(3):642-661.

16. Davies RH, Twining CJ, Cootes TF, et al. A minimum description length approach to statistical shape modeling. IEEE Trans Med Imag. 2002;21(5):525-537.

17. Zheng G, Schumann S. 3D reconstruction of a patient-specific surface model of the proximal femur from calibrated $X$-ray radiographs: a validation study (a). Med Phys. 2009;36(4):1155-1166.

18. Zheng G. Statistical shape model-based reconstruction of a scaled, patient-specific surface model of the pelvis from a single standard AP X-ray radiograph. Med Phys. 2010;37(4):1424-1439.

19. Zhu Z, Li G. Construction of 3D human distal femoral surface models using a 3D statistical deformable model. J Biomechan. 2011;44(13):2362-2368.

20. Baka N, Kaptein BL, de Bruijne M, et al. 2D-3D shape reconstruction of the distal femur from stereo X-ray imaging using statistical shape models. Med Image Anal. 2011;15(6):840-850.

21. Cerveri P, Baroni G, Confalonieri N, Manzotti A. Patient-specific modeling of the trochlear morphologic anomalies by means of hyperbolic paraboloids. Comput Assist Surg. 2016;21(1):29-38.
22. Blanc R, Seiler C, Székely G, et al. Statistical model based shape prediction from a combination of direct observations and various surrogates: application to orthopaedic research. Med Image Anal. 2012;16(6):1156-1166.

23. Dong $X$, Ballester MAG, Zheng G. Automatic extraction of femur contours from calibrated X-ray images using statistical information. J Multimedia. 2007;2(5). https://doi.org/10.4304/jmm.2.5.46-54.

24. Zheng G, Dong X, Rajamani KT, et al. Accurate and robust reconstruction of a surface model of the proximal femur from sparse-point data and a dense-point distribution model for surgical navigation. IEEE Trans Biomed Eng. 2007;54(12):2109-2122.

25. Zhu Z, Li G. Construction of 3D human distal femoral surface models using a 3D statistical deformable model. J Biomechan. 2011;44(13):2362-2368.

26. Richter PH, Steinbrener J, Schicho A, Gebhard F. Does the choice of mobile c-arms lead to a reduction of the intraoperative radiation dose? Injury. 2016;47(8):1608-1612.

27. Myronenko A, Song X. Point set registration: coherent point drift. IEEE Trans Pattern Anal Machine Intell. 2010;32(12):2262-2275.

28. Besl PJ, McKay HD. A method for registration of 3-D shapes. IEEE Trans Pattern Anal Machine Intell. 1992;14(2):239-256.

29. Jia X, Yan H, Cerviño L, et al. A GPU tool for efficient, accurate, and realistic simulation of cone beam CT projections. Med Phys. 2012;39(12):7368-7378.

30. Wicklein J, Kunze H, Kalender WA, Kyriakou Y. Image features for misalignment correction in medical flat-detector CT. Med Phys. 2012;39(8):4918-4931.

31. Cerveri P, Pedotti A, Borghese NA. Combined evolution strategies for dynamic calibration of video-based measurement systems. IEEE Trans Evolution Comput. 2001;5(3):271-282.

32. Suttorp T, Hansen N, Igel C. Efficient covariance matrix update for variable metric evolution strategies. Mach Learn. 2009;75(2):167-197.

33. Bäck T, Foussette C, Krause P. Contemporary Evolution Strategies. Natural Computing Series. Berlin: Springer; 2013.

34. Cerveri P, Marchente M, Bartels W, et al. Towards automatic computer-aided knee surgery by innovative methods for processing the femur surface model. Int J Med Robot Comput Assist Surg. 2010;6(3):350-361.

35. Li J-S, Tsai T-Y, Wang S, et al. Prediction of in vivo knee joint kinematics using a combined dual fluoroscopy imaging and statistical shape modeling technique. J Biomechan Eng. 2014;136(12):124503

36. Scholes C, Sahni V, Lustig S, et al. Patient-specific instrumentation for total knee arthroplasty does not match the pre-operative plan as assessed by intra-operative computer-assisted navigation. Knee Surg Sports Traumatol Arthrosc. 2013;22(3):660-665.

37. Chaibi $Y$, Cresson T, Aubert B, et al. Fast 3D reconstruction of the lower limb using a parametric model and statistical inferences and clinical measurements calculation from biplanar X-rays. Comput Methods Biomechan Biomed Eng. 2012;15(5):457-466.

38. Mahfouz MR, Merkl BC, Abdel Fatah EE, et al. Automatic methods for characterization of sexual dimorphism of adult femora: distal femur. Comput Methods Biomechan Biomed Eng. 2007;10(6):447-456. 